\section{TAKE THE GUESSWORK OUT OF OCCLUSION}

Indent Systems are exclusive UK distributors for T-Scan, the computerised occlusal force analysis device which takes the guesswork out of occlusion.

T-Scan enables dentists to enhance the accuracy of their diagnosis and treatment in a variety of clinical applications including implantology, periodontology and cosmetic dentistry. The patient simply bites on a thin sensor which relays changes in occlusal pressure as they move from initial contact into maximum intercuspation and excursive movements. This data is transmitted, in thousandths of a second increments, onto an easy to understand visual display.

From single unit restorations to full mouth rehabilitation, it is important for patient comfort, as well as restoration longevity, to obtain a balanced

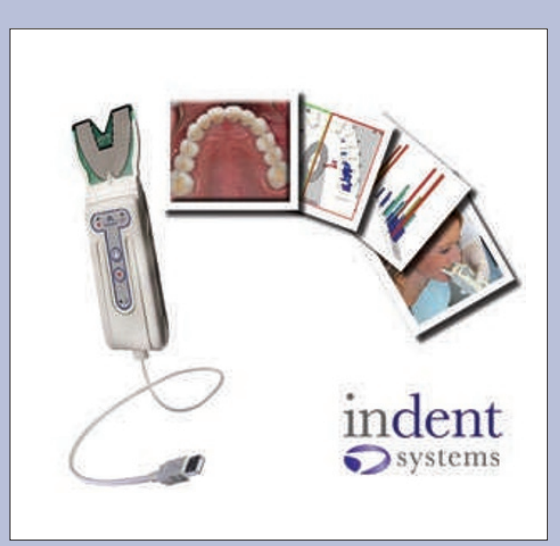

occlusion with mutual anterior/posterior support. T-Scan allows clinicians to perfect occlusal balance with accuracy and confidence and enables them to measure the force and timing of the functional bite to diagnose and treat the source of occlusal problems.

Reader response number 53

\title{
COURSES TO ENSURE YOUR PRACTICE THRIVES
}

The Dentistry Business offers a range of education courses for dentists and practice managers from one-day workshops to 10-month modular programmes, running at locations around the country from now until the end of the year.

'Starting your own practice' explores the key issues about opening and running an NHS or private practice and covers the most important actions you need to take before opening and in the early months. Acquiring an existing practice at the right price, recruiting, retaining and developing the right team and understanding the role of the CQC, clinical governance and best practice are just some of the topics covered in this highly rated course.

'Making the most of your practice' will help you recognise the solutions to some of the key business and operating challenges you face and the most important actions you need to take to ensure the practice thrives in the future. How to build loyalty and acquire the patients you want and how to expand your practice offerings, including how to add new services and what they should be, will all be explored in detail. The workshops cost
$£ 245$ for dentists and £175 for DCPs.

In addition to the above one-day workshops, The Dentistry Business also provides ten-month University accredited programmes - The Professional Certificate in Dental Practice Management and The Postgraduate Certificate in the Mastery of Dental Practice Management - both of which provide the theoretical and practical tools required to support the operation of a single or multi-site practice. the Postgraduate Certificate also provides credits for the FGDP Fellowship compulsory 'Management' section. A new series of these modular programmes will start in October 2010 and are now open for reservations.

All courses are led by the Partners in The Dentistry Business who are uniquely qualified to deliver expert advice in the field of dental practice management. Lester Ellman and Carl Parnell are both highly reputable practitioners with experience of both NHS and private practice and Sim Goldblum is a business executive with extensive corporate experience in marketing and finance.

Reader response number 54
PREVENT ACCIDENTAL SETTING OF COMPOSITES

There is always a risk that light curing materials will start polymerising in ambient light and even if it is only a slight amount, it can significantly affect their handling properties. DMG UK's LuxaTray has been specifically designed to prevent this risk.

LuxaTray is a light-shielding accessory for mixing and temporary storage of light-sensitive materials and solutions. The design of the opaque cover permits easy one-handed operation for convenient chairside use.

The sturdy, anti-skid LuxaTray is fully autoclavable. The inserts are replaceable and scratch resistant. The special shape of the wells matches the bend of the Heidemann spatula and allows for even easier handling and instrumentation.

Reader response number 55

\section{因DMG}

\section{INMAN ALIGNER TRAINING}

Specialist dental course provider Tipton Training Ltd has been selected to host the very first Inman Aligner training session in the North of England. The one-day course will allow dentists to receive full Inman Aligner accreditation and will take place at Tipton Training's headquarters in Manchester on Thursday 15 July 2010.

The Inman Aligner is an invisible brace that enables crowded or protruding front teeth to be straightened faster than ever before. Barely visible, apart from a single clear wire, the Inman Aligner can easily be slipped in and out of the mouth, allowing the wearer to go brace-free for important occasions.

Reader response number 56 